Trinity University

Digital Commons @ Trinity

Engineering Faculty Research

Engineering Science Department

2021

\title{
The Effect of Freespace Properties on Unilateral Stiffness \\ Classification
}

Emma Treadway

Trinity University, etreadwa@trinity.edu

Kristian Journet

Trinity University, kjournet@trinity.edu

Follow this and additional works at: https://digitalcommons.trinity.edu/engine_faculty

Part of the Engineering Commons

\section{Repository Citation}

Treadway, E., \& Journet, K. (2021). The effect of freespace properties on unilateral stiffness classification. 2021 IEEE World Haptics Conference, 1-6.

This Post-Print is brought to you for free and open access by the Engineering Science Department at Digital Commons @ Trinity. It has been accepted for inclusion in Engineering Faculty Research by an authorized administrator of Digital Commons @ Trinity. For more information, please contact jcostanz@trinity.edu. 


\title{
The Effect of Freespace Properties on Unilateral Stiffness Classification
}

\author{
Emma Treadway ${ }^{1}$ and Kristian Journet ${ }^{1}$
}

\begin{abstract}
Virtual environments rendered through kinesthetic haptic devices have frequency-dependent dynamic properties that affect perception. Previous studies on the perception of virtual walls have suggested that the properties not only of the wall, but also of the freespace with which it is paired, influence the perceived wall stiffness (bias) and discrimination thresholds. In this work, two experiments were undertaken to examine the effect of mass and damping properties in freespace on virtual wall stiffness classification. The results suggest that there may be alterations to classification performance under large changes in freespace conditions, but that small changes in mass and damping do not appear to significantly affect performance. These results suggest that additional study is needed to fully understand the effects of freespace properties on wall stiffness classification, which may differ from their effects on discrimination or bias.
\end{abstract}

\section{INTRODUCTION}

The dynamics of a virtual environment (VE) rendered with a haptic device are necessarily influenced by the underlying mechanics of the device used to display the VE to the user. The controller's goal is to attempt to enforce transparency [1] despite this, such that the rendered environment matches the desired VE dynamics. When rendering freespace, the physical properties of the device should be compensated for as much as possible, and the device should be designed to be as light as possible to enable this; however, when rendering a virtual wall, we should seek to maximize the magnitude of the force that the user feels. Fundamental control limitations put these two goals at odds with one another, and in practice tradeoffs must be made in selecting both the device hardware and the controller.

Across the frequency spectrum, the dynamic response displayed to the user may be thought of as a combination of effective impedance primitives such as effective stiffness, damping, and mass [2]. Thus, the study of the perception of rendered VEs is in essence the study of human perception of combinations of these mechanical primitives. Understanding how the hardware and controller properties show up in the rendered dynamics is critical because of the frequencydependent nature of human perception of combinations of these primitives [3]. A brief summary of some of the most relevant recent work in this area is given in Table I.

The effects of the presence of some mechanical primitives on the perception of others may be quantified in a number of different ways. Among the most common is the measurement of thresholds such as the just-noticeable difference (JND), often expressed as a Weber fraction (ratio of the detectable

\footnotetext{
This work was supported in part by ADVANCED Motion Controls.

${ }^{1}$ Department of Engineering Science, Trinity University, San Antonio, TX, USA etreadwa@trinity. edu
}

change to the base value): notably, recent work by $\mathrm{Fu}$ et al. suggests a frequency-dependent Weber's law for massspring-damper systems [3], [4]. These widely-used threshold measurement approaches are capable of measuring the effects in terms of the resolution of the human sensory system, but are prone to issues of response bias [5]. In addition to altering the thresholds at which differences can be felt, the presence of combined mechanical primitives in a system can create bias in the level of the property of interest; in [6], van Beek et al. explored the effects of added damping either inside a virtual object or globally (both inside and outside the virtual object), and found that damping in the presence of a contact transition decreases perceived hardness in either case, but to a greater degree when the damping is global.

While JND quantifies what level of difference can be discerned in an immediate comparison and bias reveals information about how the perceived level of a property might be skewed, many applications of haptic sensing require an ability to remember differences between physical propertiesfor example, a dentist or surgeon distinguishing healthy and unhealthy tooth or tissue, or a shopper identifying a ripe avocado. These activities rely not only on the ability to distinguish a change in physical property, but also the ability to remember and identify it. An alternative measure, the outcome of which is influenced by both the resolution of the human sensory system and memory limitations, is classification performance, which measures not only how well users can tell the difference between VEs, but how well they can remember and identify them [5].

Contact transitions in unilateral virtual environments like virtual walls can excite the higher frequencies at which unintended device behaviors influence perception; classification of stiffness was shown to be impaired by contact transitions in two previous experiments [9], [7]. Both experiments also examined the effects of rendering the VEs with high or low transmission ratio devices-in [9], this created a significant effect on classification performance; in contrast, no such effect was seen in [7]. It was postulated that this may have been due to a mismatch between the damping and mass

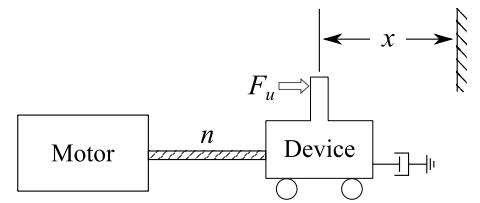

(a) Generic device schematic

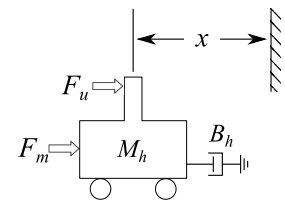

(b) Equivalent Model
Fig. 1. (a) Generic schematic for a 1-dimensional haptic interface. (b) The device and motor parameters (reflected through the gear ratio) are lumped in model parameters $M_{h}$ and $B_{h}$. Reproduced with permission from [7]. 
TABLE I

A BRIEF SUMMARY OF RECENT WORK ON PERCEPTION OF COMBINATIONS OF MECHANICAL PRIMITIVES

\begin{tabular}{|l|l|l|l|}
\hline First author & Perception of... & In the presence of... & Findings \\
\hline Rank [8] & Bilateral damping & Stiffness or mass & Damping JND increased by stiffness or mass \\
\hline Colonnese [2] & $\begin{array}{l}\text { Bilateral stiffness \& } \\
\text { damping }\end{array}$ & $\begin{array}{l}\text { Damping generated by delay or } \\
\text { filtering }\end{array}$ & $\begin{array}{l}\text { Effective impedance decomposition predicts which properties' percep- } \\
\text { tion is altered }\end{array}$ \\
\hline van Beek [6] & Unilateral stiffness & Damping (local or global) & Damping decreases perceived hardness, with larger effect when global \\
\hline Fu [4], [3] & $\begin{array}{l}\text { Bilateral stiffness, } \\
\text { damping, or mass }\end{array}$ & Stiffness, damping, and/or mass & $\begin{array}{l}\text { Extended Weber's Law predicts spring, mass, \& damper JNDs for } \\
\text { single frequency interactions }\end{array}$ \\
\hline Treadway [9], [7] & Unilateral stiffness & Damping and mass (level) & Classification performance impaired by contact transitions \\
\hline Treadway [10] & Bilateral stiffness & Damping (predictability) & Classification performance likely impaired by unpredictable damping \\
\hline
\end{tabular}

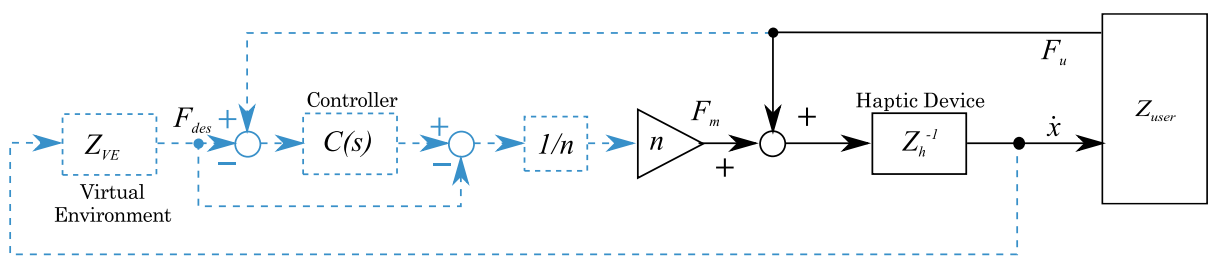

Fig. 2. Closed-loop impedance controller. Black solid arrows and outlines represent physical quantities, while blue dashed lines indicate signals and systems within the computer. The transitions from solid black to dashed blue indicate the presence of sensors (e.g., load cells and encoders), and dashed blue to solid black is the motor. Figure reproduced with permission from [7].

properties inside and outside the unilateral spring in [9] that was not present in the experiment of [7].

This study therefore seeks to understand the effect of changing only the freespace VE on the perception of the stiffness of unilateral virtual springs, measured in terms of participants' ability to classify virtual walls of different stiffness. We expect that altering the mass and damping properties of the freespace VE will alter unilateral spring classification performance for two reasons: first, local and global damping are known to have different effects on stiffness perception bias [6] - along with possible similar effects from local and global mass, this means that we expect perception to be different when mass and/or damping are local to the virtual wall or global. Second, unpredictable damping properties appear to make stiffness discrimination more difficult [10]; participants might perceive the altered mass or damping at the transition from freespace into the wall as an unexpected change in the mass or damping of the "tool" with which they are exploring springs, which would impair discrimination.

\section{Modeling And Apparatus}

A 1-dimensional kinesthetic haptic device can be modeled as shown in Fig. 1. The device has impedance $Z_{h}=$ $M_{h} s+B_{h}$, consisting of the mass $M_{h}$ and damping $B_{h}$ that include reflected properties from the motor acting through the gear ratio. Under impedance control [11], [12], the user's displacement of the device is measured, and a loop is closed to respond with a force applied by the motor as shown in Fig. 2. Its driving-point impedance response is

$$
\mathcal{Z}(s)=\frac{F_{u}}{\dot{x}}=Z_{V E}+Z_{h} /(1+C(s)) .
$$

In this work, closed-loop (CL) proportional control with $C(s)=C_{p}$ and open-loop (OL) $C_{p}=0$ control are employed. A spring VE is rendered with

$$
Z_{V E}(s)=K_{V E} / s \text {. }
$$

Described in terms of its effective impedances [2], the closed-loop driving point impedance (1) while rendering a spring (2) has an effective stiffness (ES) of $K_{V E}$ at low frequencies. As frequency increases, ES is reduced until it can no longer be rendered, and an effective mass (EM) appears instead, approaching $\frac{M_{h}}{1+C_{p}}$ (up to the Nyquist frequency where continuous approximation of the discrete dynamics break down). An effective damping (ED) of $\frac{B_{h}}{1+C_{p}}$ is also present across all frequencies.

An ideal damping VE is described as $Z_{V E}(s)=B_{V E}$. In practice, though, position is measured via an encoder and velocity must be estimated from the measured position. With a first-order lowpass-filtered velocity estimate with corner frequency $\omega_{0}$, the implementation becomes

$$
Z_{V E}(s)=B_{V E} \frac{\omega_{0}}{s+\omega_{0}} .
$$

Rendering this VE, the driving point impedance response (1) results in effective damping and mass

$$
\begin{aligned}
E D & =B_{V E}+B_{h} /\left(1+C_{p}\right) \\
E M & =M_{h} /\left(1+C_{p}\right),
\end{aligned}
$$

though the ED reverts to $B_{h} /\left(1+C_{p}\right)$ above the corner frequency of the filter.

In this work, a reproduction of the haptic device described in [7] was used. A Maxon RE65 motor is connected to the motion of a Del-Tron HPS3-4 linear slide via a 1-inch capstan. Position is measured via a US Digital encoder (E21024-315-IE-H-D-B), while forces applied at the small 3D printed handle are measured with a Transducer Techniques LSP-10 load cell, with control and sampling at $1 \mathrm{kHz}$ via Simulink Desktop Real-Time. System identification via manual excitation and least-squares fitting (methods also described in [7]) was performed in two trials. Both trials yielded $M_{h}=1.60 \mathrm{~kg}$. $B_{h}$ was more variable, measuring $21.1 \mathrm{Ns} / \mathrm{m}$ and $13.5 \mathrm{Ns} / \mathrm{m}$; an average value of $17.3 \mathrm{Ns} / \mathrm{m}$ is used for theoretical calculations. 

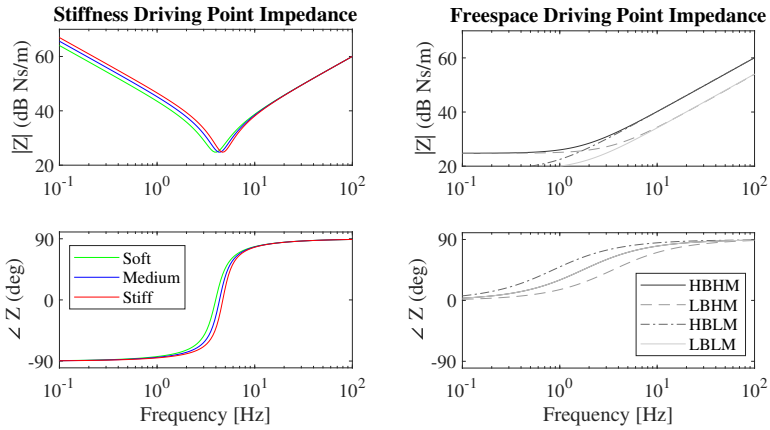

(a)
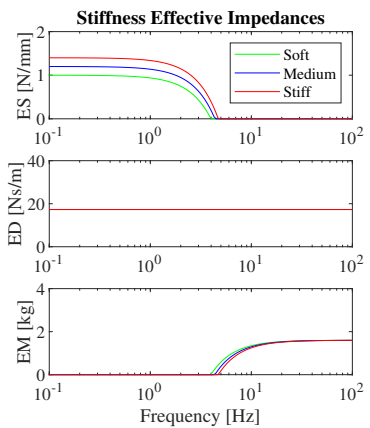

(c)

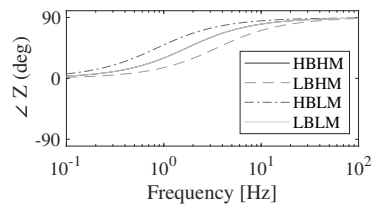

(b)
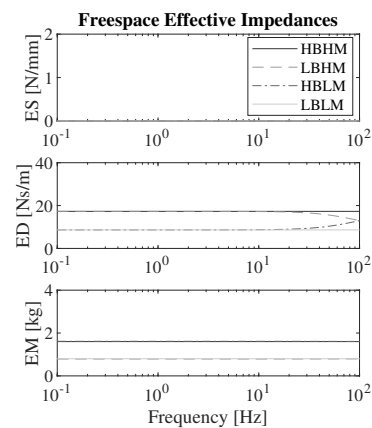

(d)

Fig. 3. Modeled behavior for the environments used in experiment 1: (a)-(b) Magnitude and phase responses inside the walls (virtual springs) and in the four freespace conditions, and (c)-(d) Effective impedance decompositions of the responses for the wall and freespace VEs.

\section{EXPERIMENT 1}

\section{A. Methods}

A forced-response classification task was employed, with three different virtual walls modeled as unilateral virtual springs of stiffness $K_{V E} \in\{1000,1200,1400\} \mathrm{N} / \mathrm{m}$, all rendered with OL impedance control $\left(C_{p}=0\right)$ and no damping $\left(B_{V E}=0\right)$ as shown in Fig. 3a and 3c. In order to understand the effects of compensation in freespace for either mass or damping, these unilateral springs were paired with a freespace VE that varied in each experimental condition. Four conditions with high and low levels of EM (1.6 or 0.8 $\mathrm{kg}$ ) and $\mathrm{ED}(17.3$ or $8.65 \mathrm{Ns} / \mathrm{m})$ in freespace were as follows; the low levels were selected to feel very different from the OL mass and damping (differences well beyond the Weber fraction, and tested with a small pilot experiment). Required levels of $B_{V E}$ and $C_{p}$ for each condition follow from (4):

1) High Damping/High Mass $(H B H M)$ : OL freespace, with $C_{p}=0, B_{V E}=0$.

2) Low Damping/High Mass ( $L B H M): C_{p}=0$ with negative virtual damping $B_{V E}=-8.65 \mathrm{Ns} / \mathrm{m}$.

3) High Damping/Low Mass $(H B L M): C_{p}=1$ with positive virtual damping $B_{V E}=8.65 \mathrm{Ns} / \mathrm{m}$ to compensate for device mass only.

4) Low Damping/Low Mass ( $L B L M)$ : $C_{p}=1$ to compensate for device mass and damping, $B_{V E}=0$.

For the velocity used to render the virtual dampers, a
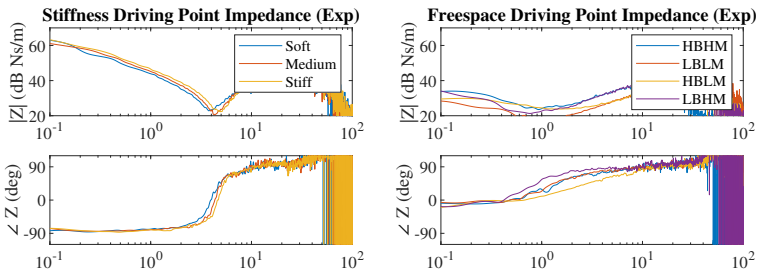

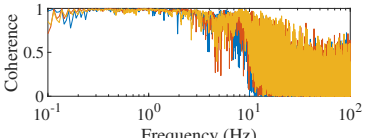

(a)
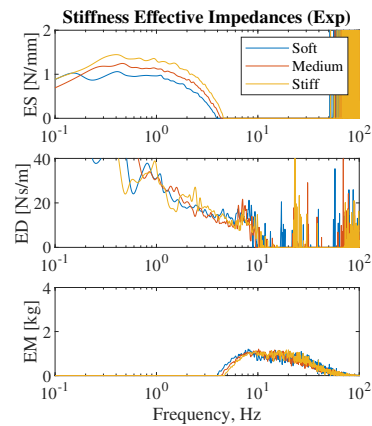

(c)

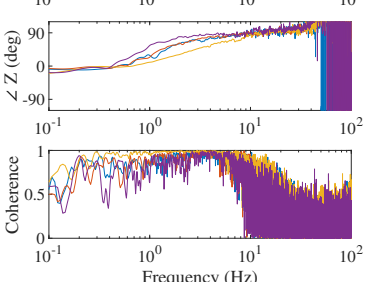

(b)
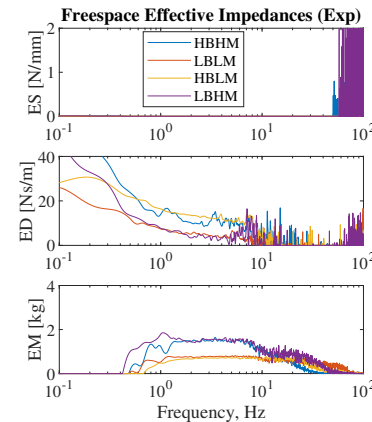

(d)
Fig. 4. Virtual wall and freespace experimental responses for experiment 1 VEs, as driving point impedances along with coherence between the input and output measurements (a-b) and effective impedances (c-d), all generated using a 10000-point Hann window. Virtual wall (a \& c) results match very well with theoretical predictions across the excited frequency spectrum (up to about $10 \mathrm{~Hz}$ ), except for ED, which varies with frequency. For the freespace ( $b \& d$ ), the viscous damping of Fig. $3 d$ does not perfectly capture the response; however, the damping in the $H B H M$ and $H B L M$ conditions match reasonably well across the frequency spectrum, as do the damping in $L B L M$ and $L B H M$.

corner frequency of $\omega_{0}=200 \pi(100 \mathrm{~Hz})$ was employed ${ }^{1}$. The predicted driving point impedance in each freespace VE can be seen in Fig. $3 b$ and $3 d$. It should be noted, however, that because viscous damping is not a perfect model of the friction in the device, it is unlikely that the damping values match perfectly in practice between the two high or two low damping conditions. Additionally, the discrete implementation means that the continuous responses predicted in Fig. 3 will break down as the Nyquist frequency $(500 \mathrm{~Hz})$ is approached. Experimental responses are shown in Fig. 4.

Nine participants (mean age 21.8 years, all right-handed, 5 female) including both authors participated under an experimental procedure approved by the Trinity University Institutional Review Board. Due to COVID-19 precautions, recruitment was limited to individuals already participating in research in the lab; however, most were naive to the details of the experiment at the time they participated, and all were naive to the order of conditions during the experiment.

During each trial, participants wore noise-cancelling headphones playing pink noise to block auditory cues, and sat at the device with a divider blocking their view of the

\footnotetext{
${ }^{1}$ This is well above both the frequency range humans can excite and the range observed in [7] when tapping on a virtual wall.
} 


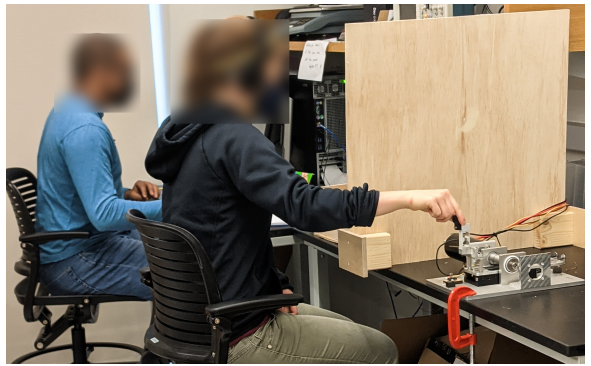

Fig. 5. Participants' view was blocked by a divider, and pink noise was played through noise-canceling headphones as the randomized walls were presented during the experiment.

device and their hand, as shown in Fig. 5. Participants were instructed to grasp the handle with the thumb and forefinger of their dominant hand and to tap on the virtual walls (pulling towards themselves), always feeling them by transitioning from freespace to the wall; participants' strategies were otherwise unconstrained ${ }^{2}$. At the beginning of each trial, participants were exposed to the three different virtual walls rendered along with the condition's particular freespace VE: the soft, medium, and stiff walls were presented in order and identified to the participants at least twice, and the participants could request to feel them as many more times as they wished before starting the trial. Then, 46 randomized walls were presented ${ }^{3}$, and participants verbally reported their identification of each as soft, medium, or stiff. The order of trials was randomized for each participant.

Upon inspection of the results, it was discovered that two participants (S6 and S7) had unusual artefacts (abrupt spikes) in the recorded force data from their tests, which would have influenced the rendered VE in the CL conditions. S7 was able to come repeat the test in a session that did not display those artefacts; S6 has been excluded from the analysis, so results from $N=8$ participants are presented.

\section{B. Results}

Performance on the classification task was evaluated with two metrics. The percent correct answers gives an indication of how well participants can identify springs throughout the task. The information transfer (IT) measures how many distinct levels of stimulus a participant can identify [5], [13]. Both metrics are examined as they are complementary: a participant could have perfect distinction between all 3 levels of stimulus, but have them all reversed, which would yield low percentage correct but high IT. Conversely, a participant's percent correct could be artificially high because they always guessed "stiff" and happened to be in a trial where randomization presented many stiff springs, but IT would be low.

Averaged results for each condition are shown in Fig. 6 as confusion matrices, with the percent correct answers and the IT (in bits) for each condition reported above the matrix. The confusion matrices indicate how frequently the answer

\footnotetext{
${ }^{2}$ Participants self-selected speed and number of times they felt each wall.

${ }^{3}$ At least $5\left(3^{2}\right)=45$ trials are required with stiffness three levels to minimize bias in IT estimation [5].
}

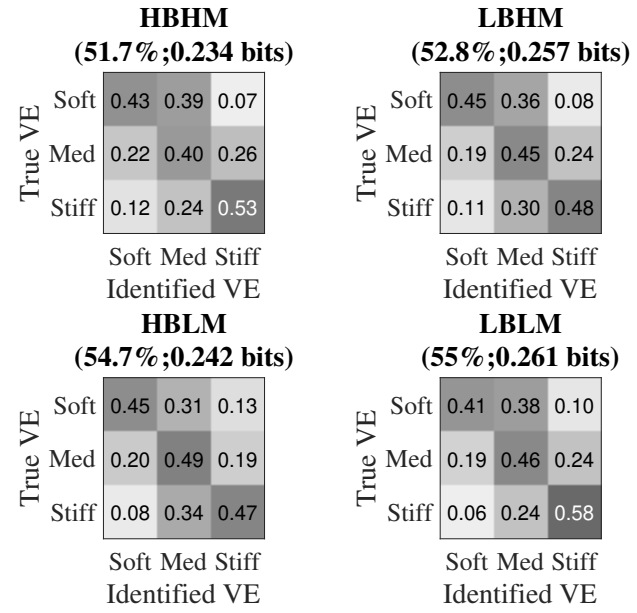

Fig. 6. Classification results for experiment 1, averaged across participants (excluding S6). Average percent correct and IT are reported above each averaged confusion matrix.
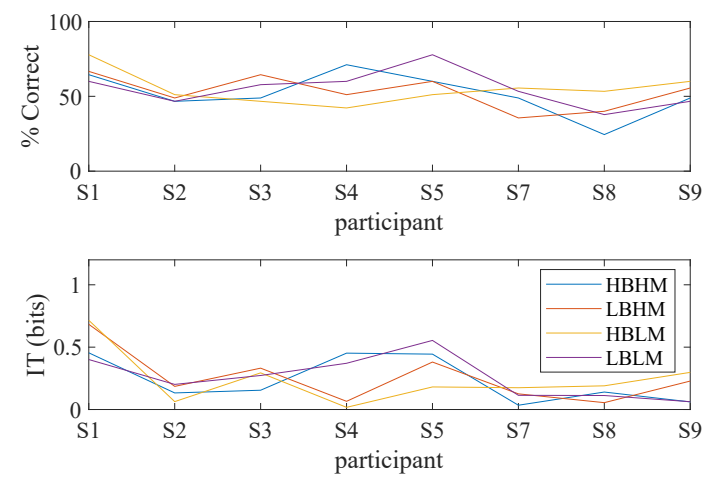

Fig. 7. Experiment 1 classification metrics by participant: percentage of walls correctly identified (top) and information transfer (bottom).

soft/medium/stiff (columns) was given when the true spring (rows) was presented. Perfect performance would appear as solid black squares (1.00) in the boxes which intersect the columns and rows of the same name; all other boxes would be white $(0.00)$. The minor color variations seen in these results between the diagonal and off-diagonal elements indicate a large amount of confusion among the three springs across all conditions.

Each participant's individual performance is summarized in Fig. 7. Subject-to-subject variations were stronger than any trends across conditions. A 2-way between-subjects (repeated measures) analysis of variance (RMANOVA) confirms that there were no significant effects, either by damping or mass level outside the virtual wall, and no significant interaction effects ( $p>0.3$ with $F(1,7) \leq 0.92$ for each).

To investigate whether the freespace VE impacted the exploration strategy, we calculated the wall contact velocities (measured by filtering a first difference estimate with a firstorder $100 \mathrm{~Hz}$ cutoff frequency lowpass filter, and extracting the instants at which the wall was crossed). Differences in velocity have potential to influence the rate-hardness or extended rate-hardness metrics that are tied to perception of wall hardness [14], [15]. For each participant, the average 


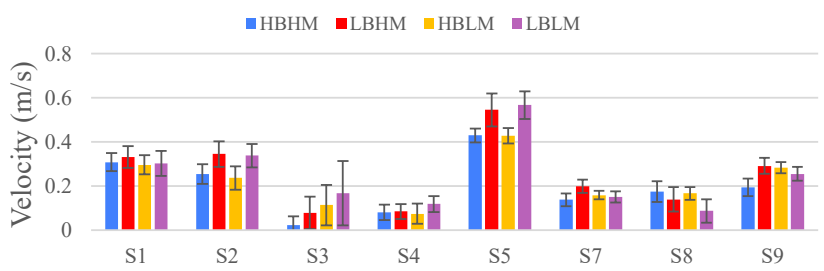

Fig. 8. Average contact velocity across all taps in each condition by participant for experiment 1 ; error bars indicate \pm 1 standard deviation.

and standard deviation of contact velocity were calculated for each trial across all taps, and are shown in Fig. 8. Two-way RMANOVA does not reveal significant effects by damping $(F(1,7)=4.19, p=0.08)$, mass level $(F(1,7)=$ $0.35, p=0.57)$, or interaction effect $(F(1,7)=1.42, p=$ 0.27 ) for average speed; for speed variability, only damping approaches significance $(F(1,7)=5.07, p=0.06)$, while mass $(F(1,7)=0.42, p=0.54)$ and interaction effects $(F(1,7)=0.26, p=0.63)$ did not significantly influence the variability of contact velocity.

\section{EXPERIMENT 2}

The lack of significant differences or recognizable trends in the results of experiment 1 may have been due to one of several reasons. First, spring constants may have been chosen too close to the discrimination threshold, such that poor performance overall washes out any trends. Second, the stiffness values used in [9] and [7] were much higher; it is possible that the effects of freespace conditions are not significant in this stiffness range. Third, a larger difference in mass and damping inside and outside the virtual wall may be needed to affect classification performance. Or, finally, mass and damping outside of a virtual wall may have no significant effect on stiffness classification performance. While no experiment can confirm the null hypothesis (last possibility), we asked participants to return a second time to perform a modified experiment addressing the first three points. In order to widen the gap between the low and high levels of mass and damping, closed-loop impedance control was used in both cases, but with a negative gain in the highmass cases (as well as inside the unilateral spring VEs).

\section{A. Methods}

The experimental procedures were the same as in experiment 1 , but the conditions were modified. The controller gain and VE damping values were adjusted in three of the four experimental conditions to increase the differences between the low and high values of mass and damping. These conditions created high/low damping values of 34.6/8.65 $\mathrm{Ns} / \mathrm{m}$ and high/low mass values of $3.2 / 0.8 \mathrm{~kg}$, as shown in Fig. 9. The freespace conditions are:

1) $H B H M: C_{p}=-0.5, B_{V E}=0$.

2) $L$ BHM: $C_{p}=-0.5, B_{V E}=-25.95 \mathrm{Ns} / \mathrm{m}$.

3) $H B L M: C_{p}=1, B_{V E}=25.95 \mathrm{Ns} / \mathrm{m}$.

4) $L B L M: C_{p}=1, B_{V E}=0$.

The three virtual walls were given stiffness values $K_{V E} \in$ $\{3000,5000,8333\} \mathrm{N} / \mathrm{m}$, matching those used in $[9]^{4}$, and

\footnotetext{
${ }^{4}$ All stiffness values are still low enough to achieve passivity [16].
}

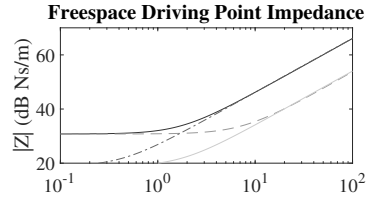

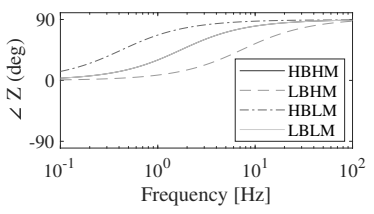

(a)

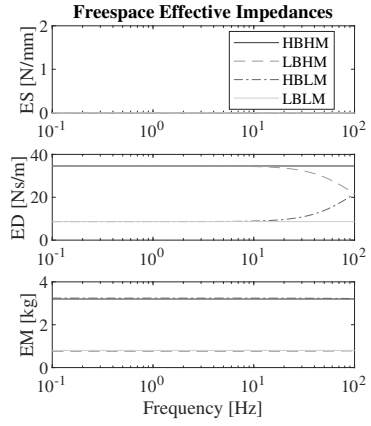

(b)
Fig. 9. Freespace VEs for Experiment 2, as (a) driving point impedance and (b) effective impedances.

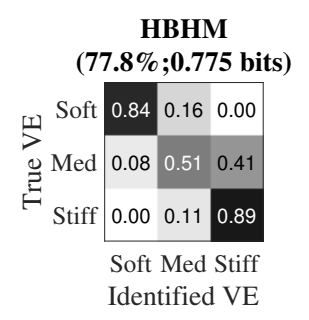

HBLM

(71.1\%;0.654 bits)

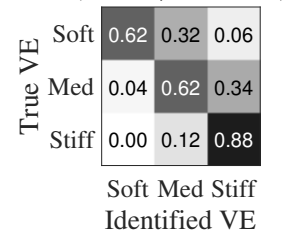

LBHM

(67.2\%;0.594 bits)

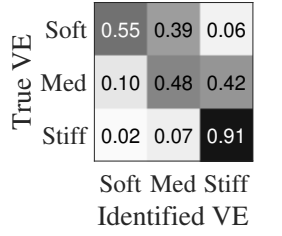

LBLM

(70\%;0.711 bits)

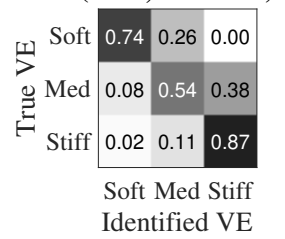

Fig. 10. Classification results for experiment 2, averaged across participants, as confusion matrices, percent correct, and IT.

were rendered with $C_{p}=-0.5$ to match the high mass and damping values in the freespace conditions. Four participants completed Experiment 2, including both authors (all experiment 1 participants were invited to participate, excluding S6 and $\mathrm{S} 7$ who had already been invited back once as allowed per our IRB-approved protocol).

\section{B. Results}

Summary results from experiment 2 are shown in Fig. 10. Overall, the levels of performance (both IT and percent correct) are higher in this new experiment, indicating that we were successful in making the task easier to avoid overall task difficulty masking differences between conditions (or, potentially, extra experience improved participants' performance, making this task easier the second time).

It is notable that the differences between conditions are more pronounced in this second experiment. While our study did not include a survey, some participants spontaneously reported that certain conditions made them feel less confident about their answers than others. Interestingly, though, some of the effects seem to be different for different people, as indicated by Fig. 11. A small but consistent trend across all four participants is that percent correct in the HBHM condition matched or exceeded percent correct in LBLM (blue 

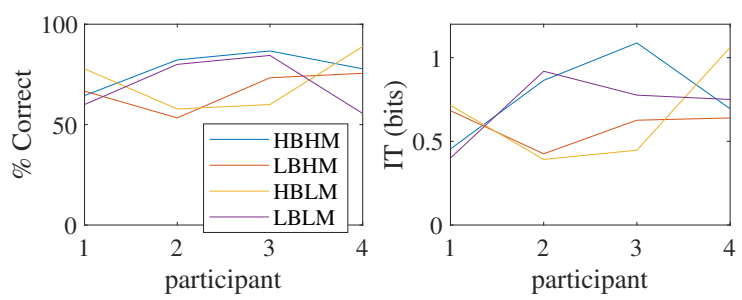

Fig. 11. Experiment 2 performance metrics for each participant.

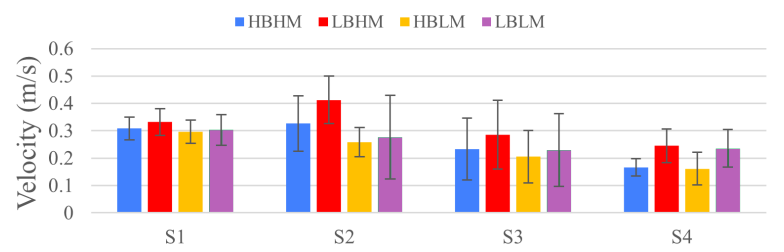

Fig. 12. Average contact velocity in each condition by participant for experiment 2; error bars indicate \pm 1 standard deviation.

vs. purple). The trend in percent correct is also accompanied by an increase in both wall contact speed and its variability (larger standard deviation) in the low damping conditions as compared to the high damping ones across all participants, as seen in Fig. 12. Closer inspection of the data reveals that trials with large variability (e.g., all of S3's trials) tend to include many "rebound" hits on the wall at lower speeds.

\section{Discussion AND CONCLUSIONS}

Based on previous studies about perception of stiffness, we expected that manipulating levels of mass and damping in the freespace outside of a virtual wall would affect participants' ability to classify virtual walls according to their stiffness. In experiment 1 , we found no significant differences in performance caused by the freespace VEs; for most participants, the classification performance across the different freespace conditions was almost the same, particularly when looking at IT. Based on these results, we conclude that if the level of damping and mass outside of a virtual wall has an impact on classification performance, its effect is small at the levels present in this experiment (the device's OL levels vs. CL compensated levels). This motivated the design of our second experiment, which was designed to widen the gaps between conditions. The small sample size in experiment 2 precludes statistical analysis; however, performance differences between conditions were more pronounced than in experiment 1. A small but consistent trend showed that the percentage of correct answers was higher in the HBHM case than the LBLM case, and increased variability in the speed with which participants hit the wall in LBLM may be responsible for this difference.

These trends suggest that further exploration of the effects of mass and damping in freespace on wall stiffness perception is required before concluding that they do not impact classification performance. This study investigated mass and damping at or below the level inside the virtual wall; effects may be more pronounced when these levels are reversed, or when the gap between the low and high levels of mass and damping are increased even more (the study in [6] used 5 and
$30 \mathrm{Ns} / \mathrm{m}$ to study bias, which is a slightly larger gap than in our experiment 2). Incorporation of a perceptual model in the future may yield further insights into the levels of mass and damping that will affect classification performance. Additionally, a truer rendering of a viscous damper achieved through a more complex controller than simple proportional control may influence results.

\section{ACKNOWLEDGMENTS}

Thanks to Shadae Bokaye-Yiadom and Brent Gillespie for previous collaboration that raised some of these questions.

\section{REFERENCES}

[1] D. Lawrence, "Stability and transparency in bilateral teleoperation," IEEE Transactions on Robotics and Automation, vol. 9, no. 5, pp. 624-637, 1993.

[2] N. Colonnese, A. F. Siu, C. M. Abbott, and A. M. Okamura, "Rendered and characterized closed-loop accuracy of impedance-type haptic displays," IEEE Transactions on Haptics, vol. 8, no. 4, pp. 434-446, 2015.

[3] W. Fu, M. M. Van Paassen, and M. Mulder, "Human Threshold Model for Perceiving Changes in System Dynamics," IEEE Transactions on Human-Machine Systems, vol. 50, no. 5, pp. 444-453, 2020.

[4] W. Fu, A. Landman, M. Van Paassen, and M. Mulder, "Modeling human difference threshold in perceiving mechanical properties from force," IEEE Transactions on Human-Machine Systems, vol. 48, no. 4, pp. 359-368, 2018.

[5] L. A. Jones and H. Z. Tan, "Application of psychophysical techniques to haptic research," IEEE Transactions on Haptics, vol. 6, no. 3, pp. 268-284, 2013.

[6] F. E. van Beek, D. J. F. Heck, H. Nijmeijer, W. M. B. Tiest, and A. M. Kappers, "The effect of global and local damping on the perception of hardness," IEEE Transactions on Haptics, vol. 9, no. 3, pp. 409-420, 2016.

[7] E. Treadway and R. B. Gillespie, "Unilateral and Bilateral Virtual Springs: Contact Transitions Unmask Device Dynamics," IEEE Transactions on Haptics, vol. 12, no. 2, pp. 205-216, 2019.

[8] M. Rank, T. Schauß, A. Peer, S. Hirche, and R. L. Klatzky, "Masking effects for damping JND," in Haptics: Perception, Devices, Mobility, and Communication. EuroHaptics 2012. Lecture Notes in Computer Science, vol 7283, P. Isokoski and J. Springare, Eds. Berlin, Heidelberg: Springer, 2012, pp. 145-150.

[9] E. Treadway and R. B. Gillespie, "The impact of high-frequency haptic device behavior on perception," in IEEE Haptics Symposium. San Francisco: IEEE, 2018, pp. 52-57.

[10] E. Treadway, S. Cutlip, and R. B. Gillespie, "Haptic Scene Analysis: Mechanical Property Separation Despite Parasitic Dynamics," in Haptics: Science, Technology, and Applications. EuroHaptics 2018. Lecture Notes in Computer Science, D. Prattichizzo, H. Shinoda, H. Tan, E. Ruffaldi, and A. Frisoli, Eds., vol. 10893 LNCS. Pisa: Springer International Publishing, 2018, pp. 234-245.

[11] N. Hogan, "Impedance control: An approach to manipulation: Part IIIapplications," Journal of Dynamic Systems, Measurement and Control, Transactions of the ASME, vol. 107, no. 1, pp. 17-24, 1985.

[12] M. Mihelj and J. Podobnik, Haptics for Virtual Reality and Teleoperation (Vol. 64, Intelligent Systems, Control and Automation: Science and Engineering). Springer, 2012.

[13] H. Z. Tan, S. Choi, F. W. Lau, and F. Abnousi, "Methodology for maximizing information transmission of haptic devices: A survey," Proceedings of the IEEE, vol. 108, no. 6, pp. 945-965, 2020.

[14] D. A. Lawrence, L. Y. Pao, A. M. Dougherty, M. A. Salada, and Y. Pavlou, "Rate-hardness: a new performance metric for haptic interfaces," IEEE Transactions on Robotics and Automation, vol. 16, no. 4, pp. 357-371, 2000.

[15] G. Han and S. Choi, "Extended rate-hardness: A measure for perceived hardness," in EuroHaptics, A. K. et Al., Ed., vol. 6191 LNCS, no. PART 1, Amsterdam, 2010, pp. 117-124.

[16] J. J. Abbott and A. M. Okamura, "Effects of Position Quantization and Sampling Rate on Virtual-Wall Passivity," IEEE Transactions on Robotics, vol. 21, no. 5, pp. 952-964, 2005. 